\title{
RESTORING THE CARDIAC CONTRACTILE FUNCTION USING IMPLANTS WITH NEURAL BUNDLES IN THE SINOATRIAL NODE OF THE HEART IN DILATED CARDIOMYOPATHY AT DOGS
}

\author{
Vasiliu C.C. Cătălina Victoria, DVM PhD \\ Faculty of Veterinary Medicine Bucharest, USAMV, Romania
}

\begin{abstract}
The survey deals with a diagnostic method of dilated cardiomyopathy in dogs, related to a new method of treatment. Dilated cardiomyopathy is the main disease causing death in large dog breeds, having genetic determinism and palliative methods of treatment. The disease consists in the dilation of the cardiac muscle and the progressive decreasing of the heart contraction strength. The hypothesis of the treatment, which offers hope to be revolutionary, is based on the histological similarity between nodal cells and neurons from the brain. The treatment is in a theoretical phase and consists in using culture neurons in order to supply the contractil function and the cardiac automatism.
\end{abstract}

\section{Introduction:}

Dilated cardiomyopathy is a progressive ${ }^{1}$ disease of the myocardium, the dilatation initially concerning the left ventricle ${ }^{2}$, but it extends to all cardiac cavities, being accompanied by alterations of ventricular kinetics and arrhythmias ${ }^{3}$. Dilated cardiomyopathy is the most common of chronic cardiomyopathies ${ }^{1}$. It is demonstrated the predisposition of large dog breeds towards dilated cardiomyopathy (Doberman, Pincher, Great Dane, Cane Corso, Presa Canario, Bulldog American, Leonberg, Bullmastiff, Mastiff Tibetan, Schnautzer ${ }^{26}$, Irish wolfhound ${ }^{19}$ ) and the genetic transmission of this disease ${ }^{4}$.

Currently applied treatment regimens are symptomatic of secondary heart failure and arrhythmias, as an adjuvant or in a prophylactic way $^{6}$. There is no form of treatment for in situ changes ${ }^{5}$.

The purpose of this article is to present a new concept for the treatment of dilated cardiomyopathy, dealing with the contractile dysfunction of the heart ${ }^{6}$. Developing and testing this hypothesis raise tremendous possibilities for the future, including the adaptation to other cardiac diseases or human medicine. In order to be able to compensate for the contractile function, it is necessary to thoroughly study the structure that naturally deals with this - the nodal system. Cardiac tissue regeneration attempts using human induced pluripotent stem cell (hiPSC) ${ }^{18}$ or cellular cardiomyoplasty ${ }^{15}$ using stem cells residing in the heart are known.

The study presents current attempts to restore the cardiac contractile function ${ }^{6}$ and brings to light a new hypothesis: the use of culture neuronal cells ${ }^{7}$ to supplement the nodal system ${ }^{5}$ and stem cells to replace the decompensated myocardiocytes $^{1}$ in the cardiac dilatation process.

The treatment hypothesis is based on the histological resemblance ${ }^{8}$ of nodal cells to neurons in the encephalus 9 . The method of treatment is in an early phase, the theoretical one, and cumulates aspects and hypotheses of existing studies to establish the most effective technique in restoring the cardiac contractile function in $\operatorname{dog}$ affected by dilated cardiomyopathy.

\section{Materials and Methods}

The treatment consists in the use of culture neurons $^{54}$ to compensate the contractile function $^{6}$, the cardiac automatism ${ }^{10}$ and autologous stem cells by creating patient specific hiPSC ${ }^{18}$ systems. The clinical translation ${ }^{15}$ requires the isolation of stem cells 
from surgical or biopsy samples ${ }^{11}$, ex vivo expansion, and relocation ${ }^{19}$ in the affected heart. Stem cells - the highest rate of success was achieved in the monostratum approach using Roswell Basal Medium (RPMI), ascorbic acid, recombinant human albumin and Wnt pathway inhibitors ${ }^{18}$. After obtaining functional myocardiocytes in vitro ${ }^{19}$, from stem cells, relocation to the left ventricular myocardium can be achieved by ecoguided puncture ${ }^{22}$ through the transverse wall of the ribs ${ }^{14}$. Cultures of neuronal cells - maintenance on modified polypeptide media (PNW) in microfluidic system $^{15}$. They will be transplanted by injection ${ }^{33}$ into the sinoatrial node. The recipient organism of the two cell types, respectively the dog with dilated cardio-myopathy ${ }^{24}$, requires preoperative preparation ${ }^{12}$ and complex induced immunosuppression ${ }^{15}$, to avoid the immune reaction of graft rejection ${ }^{34}$.

It is necessary to use the flow cytometry for sorting cells according to the markers expressed on the surface $e^{45}$, the selection of neurons ${ }^{54}$ which have the capacity to bind to nodal cells and myocardiocytes multiplied in vitro ${ }^{35}$, with the highest cardiac regeneration potential ${ }^{36}$.

The biopsy puncture has made numerous contributions $^{12}$ to the knowledge of the structural aspects of myocardial fibers in chronic cardiomyopathies ${ }^{14}$. It was possible to establish the localization, nature and dynamics of structural and ultrastructural changes ${ }^{14}$ in chronic cardio-myopathies and the correlation with the clinical signs ${ }^{16}$.The study proposes using the same type of puncture in the sense of introducing reconstructive elements to the myocardium.

\section{Justification of the theoretical concept}

Generally, the pathology of the heart in veterinary medicine has a high frequency and involves major therapeutic problems. Cardiospheres ${ }^{15}$ or stem cells obtained directly from the heart of the patient provide a potential source for myocardial regeneration therapy, lowering the degree of immunosuppression required for the intervention ${ }^{36}$.

The cardiac dilatation process leads to overcoming the adaptive functions of the myocardium $^{6}$. Its response consists in the elongation of supra modum myocardiocytes ${ }^{7}$ to functional disability or destruction ${ }^{13}$. It is necessary to replace them and ensure a rate of gradual replacement ${ }^{8}$ of the destroyed ones, by the hiPSC systems ${ }^{44}$. These can be adapted on the basis of cardiospheres ${ }^{40}$ to increase the compatibility with the myocardium at implantation ${ }^{17}$ and the rate of transformation of these stem cells into adult myocardiocytes.

For the restoration of the nodal tissue, a cell with functional capabilities and a nodal cell-like structure is required. The neuron is, in turn, a cell with electric potential ${ }^{21}$ and its adaptability to the surface markers ${ }^{27}$ of other cells is known ${ }^{54}$. These aspects support the study hypothesis and make the neuron the perfect candidate for restoring the cardiac contraction function.

The nodal tissue (or the excito-conductive system of the heart) is considered to be part of the myocardium ${ }^{1}$, although the nodal cells are very different from the myocardial muscle fiber $^{2}$; the nodal tissue is characterized by the preservation of the embryonic self-contractile properties $^{27}$.

The excito-conductive system of the heart is a complex of highly specialized cells ${ }^{3}$ with the role of generating rhythmic excitations and of leading them into the myocardium, providing rhythmical and continuous contraction of the heart ${ }^{4}$. It consists of a dispersed portion - the Purkinje network ${ }^{1}$ represented in the form of a fine subendocardial network and a dense voluminous portion ${ }^{2}$. The agglomerations form the sinoatrial node 4 , located in the wall of the right atrium and the atrioventricular node, located at the base of the interatrial septum ${ }^{5}$. The atrioventricular node continues, without a precise demarcation, with the atrioventricular bundle.

The nodal tissue consists of 3 cell types: $P$ or the pacemaker - it produces the electric impulse, $\mathrm{T}$ - it transmits and propagates the pulse produced by the P cell and the Purkinje cell - it has the role of stopping the access of premature or ectopic electrical impulses. $P$ cells show branches of the cell membrane and form anastomoses with adjacent cells ${ }^{7}$, making electron exchanges at this level $\left(\mathrm{Ca}^{+}, \mathrm{Na}^{+}, \mathrm{K}^{+}\right)$. The potential for action spreads from one myocardial cell to the other through intercalated $\operatorname{discs}^{4}$. 

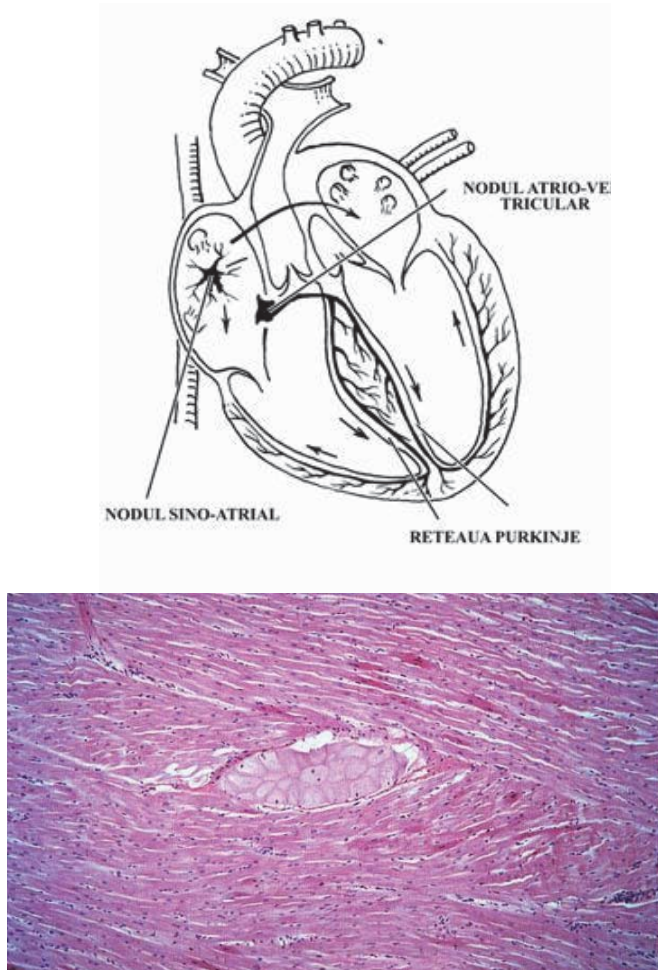

Figure 1. The histostructure of the nodal tissue vs. Organizing the nodal tissue in the heart (scheme). The HE colour was used for the preparation, the cells are larger than the myocardiocytes, and they are slightly coloured (at x60 magnification).

A particular feature of the cardiac muscle excitability is the long duration of action potency of 100-250 $\mathrm{msec}^{21}, 100$ times more than the potential of the skeletal muscle, as it is determined by the change in the membrane permeability for potassium, sodium and calcium ions $^{8}$.

The duration of a contraction is almost equal to the duration of the action potency ${ }^{12}$, thus equal to the duration of the refractory period ${ }^{32}$. Due to the long refractory period, the cord cannot sustain a continuous contraction and prevents the fusion and summation of the cardiac contractions (phenomena encountered in the skeletal muscle) $)^{27}$. This provides the time to relax the myocardium ${ }^{23}$ and to refill the cavities with blood, between systole . $^{6}$

The existence of the refractory period, also known as the "law of periodic inexcitability of the heart," explains the impossibility of tetanus occuring in the myocardium. The cardiac muscle does not respond to stimuli during the absolute refractory period. If stimulus are applied during the period of excitability ${ }^{4}$, before the physiological nodal stimulus, they can cause premature depolarization of the heart muscle ${ }^{17}$, with interruption of the heart relaxation and the appearance of a systole outside the normal rhythm - an extrasystole ${ }^{6}$. This prevents, through its own refractory period ${ }^{3}$, the cardiac response to the next sinus stimulus, which is why the extrasistole is followed by a prolonged rest, the myocardium contracting to the next sinus stimulus ${ }^{4}$.

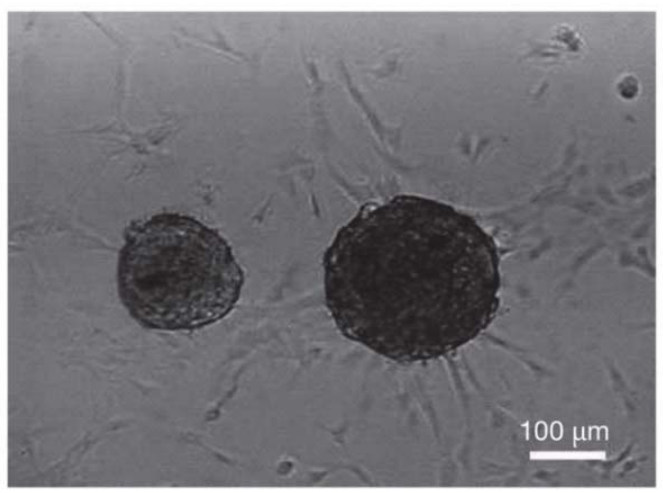

Figure 2 | Morphology of human cardiospheres. Isolated by myocardial biopsy and grown in vitro. The cardiospheres in the image have 5 days after harvesting and form an adherent monolayer.

The potential for action generated by the cells of the sinoatrial node propagates in the atrial mass at a speed rate of $0.3-0.5 \mathrm{~m} / \mathrm{sec}^{32}$. Atrioventricular node cells ${ }^{22}$ represent the only functional link between atria and ventricles ${ }^{27}$. They are separated by a layer of connective tissue that does not produce or drive potential action $^{5}$, so an insulator ${ }^{25}$. The speed rate of propagation of action potentials through the atrioventricular node is only $0.02-0.05 \mathrm{~m} / \mathrm{sec}^{32}$. The reduced speed ensures the temporal gap between the atrial and ventricular contractions ${ }^{5}$, respectively the end of the atrial systole before the ventricular systole begins ${ }^{6}$.

The cellular cardiomyoplasty or the grafting of cardiomiocytes ${ }^{15}$ is an attempt to regenerate the affected myocardium ${ }^{33}$, bringing a new hypothesis. The mammalian cord is in a continuous state of cellular fluctuation ${ }^{34}$ and has 
intrinsic regenerative potential, but it is limited $^{23}$. In adulthood, the myocardium also stores small populations of multipotent cardiacprogenitor cells ${ }^{35}$, identified by surface expression of the receptor for stem cell factor (ckit), P-glycoprotein, and the antigen stem cells 1 $(\text { Sca-1 })^{35}$. Cardiac stem cells were identified using flow cytometry ${ }^{36}$. Cells expressing Sca-1 and not expressing CD31 had the greatest potential for differentiation ${ }^{37}$.

A distinct population of cardiac stem cells was isolated in the adult rat heart. These primitive cells do not express markers for blood lines and

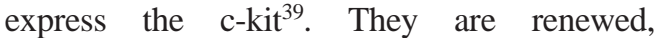
multipotent by cloning ${ }^{40}$, and have the ability to generate myocardiocytes, smooth muscle cells and endothelial cells in vitro (in the presence of $10 \%$ fetal calf serum and dexamethasone) and in vivo ${ }^{15}$

They were injected into the border area 5 hours after the induction of myocardial infarction in adult rats ${ }^{15}$, and they induced the formation of myocardiocytes, capillaries and arterioles, in the infarct area ${ }^{35}$.

The improvement of the cardiac dysfunction was found 5 weeks after the infarction ${ }^{15}$. A Sca$1^{15}$-like protein was detected by confocal microscopy and Western blot tests in human and dog myocardiocytes ${ }^{36}$. Like Sca-1, it belongs to the Ly6 family of surface antigens and it is still unarrayed ${ }^{15}$. A population of cardiac stem cells having the Isl-1 transcription factor ${ }^{15}$ has been described; they have shown an extremely efficient conversion to adult myocardiocytes in the absence of cell fusion ${ }^{40}$. However, up to this point, they have been detected only in neonatal samples in humans and rats ${ }^{15}$.

Residual cardiac stem cells are involved in the slow replacement of myocardiocytes ${ }^{7}$, replacing cells lost occasionally ${ }^{8}$ but inaccessible to the rapid regeneration of a large number of cells in response to lesions such as myocardial infarction ${ }^{15}$. In addition, bone marrow stem cells have been found to undergo a process of mobilization towards the cord after the myocardial infarction ${ }^{33}$. However, in myocardial damage, the lymphoproliferative response produces a fibrous tisue. The isolated cells form groups called cardiospheres ${ }^{15}$, in the suspensions of cultures. They mimic the biological situation in vivo when incorporated in a three-dimensional environment ${ }^{40}$, they develop upper-order intercellular structures facilitating proliferation, differentiation and angiogenesis ${ }^{15}$. The cardiospheres extended as adherent monolayer ${ }^{15}$ were transplanted to mice with severe induced immunodeficiency in the pre-infarcted $\operatorname{area}^{41}$. The replacement of the necrotic tissue with neoformation myocardiocytes and the functional improvement of the myocardium occurred within 18-20 days ${ }^{15}$.

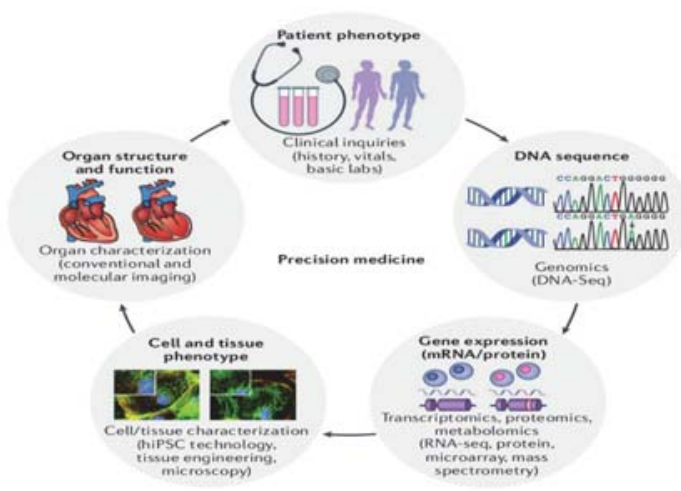

Figure 3 The role of hiPSC systems, integrated in humans. Obtaining pluripotent stem cells specific to the individual phenotype by gene expression and DNA sequence analysis will correct the structure of the modified organs regardless of the nature of the disease.

There are attempts, quoted in literature (Chen I. Yan, 2016), regarding the use of pluripotent stem cells in cardiovascular disease called hiPSC (human induced pluripotent stem cell) ${ }^{18}$. The possibilities were unlimited, the cells were reprogrammed and differentiated into several types of cardiovascular cells ${ }^{19}$ : myocardiocytes, vascular endothelial cells and smooth muscle cells corresponding to the arterial mean ${ }^{42}$. At the time, a revolutionary approach was the use of stem cells of the patient, depending on the genetic variability of the individual and their own genetic markers ${ }^{43}$.

The hiPSC differentiation protocol in myocardiocytes is based on the use of embryoloid organisms $(\mathrm{EB})^{44}$. These are mouse embryonic fibroblasts, irradiated and grown in suspension culture, where they form spontaneous cell aggregates ${ }^{45}$. The obtained cells resemble the early-stage myocardiocytes with respect to the myofibrillar organization and catecholamines response ${ }^{46}$. These can be 
structurally and electro-physiologically differentiated $^{50}$. Subsequently, the hiPSC system has been used in diseases such as: arrhythmias, cardiomyopathies, valvulopathies, blood vessel diseases, cardio-metabolic disorders ${ }^{51}$, but it cannot be used on a large scale. Major issues were related to low efficiency and considerable variability ${ }^{18}$, including in terms of aggregate size.

The next evolutionary step was the creation of enzyme-adapted cultures ${ }^{49}$, selected from the point of view of size by centrifugation ${ }^{18}$. These have resulted in uniform aggregates having a consistent cardiac differentiation ${ }^{52}$.

The precise modulation of the mesodermal induction ${ }^{53}$ was achieved by timely introducing and removing the growth factors that influenced four major pathways of development: the pathway of the bone morphogenetic protein (BMP), the beta factor or the nodal pathway, the Wnt pathway and the pathway of the Fibroblast growth factor $(\mathrm{FGF})^{18}$. Dubois and his colleagues $^{47} \quad$ achieved $98 \%$ purity myocardiocytes using an EB approach based on the sequential addition of multiple growth factors (BMP4, VEGF, activin A and DKK1 protein) followed by a flow cytometry purification, against a new patient-specific cell surface ${ }^{47}$.

The differentiation was confirmed by the position of the troponin $\mathrm{T}$ and cardiac markers ${ }^{47}$. The complexity of the technique and the intensive work required by the protocol has prevented the use of the method on a large scale. Laflamme and co-workers ${ }^{48}$ cultivated embryonic stem cells as monolayers and induced the sequential addition of cardiac differentiation and the subsequent elimination of activin $\mathrm{A}$ and BMP4; the purity of cultures was only $30 \%{ }^{48}$. Burridge and co-workers ${ }^{53}$ improved and simplified the monolayer approach using Roswell basal medium (RPMI), ascorbic acid, recombinant human albumin and Wnt pathway inhibitors, thus obtaining $95 \%$ purity myocardial fibers differentiated into 11 cell lines ${ }^{53}$. As far as in vitro neuron cultivation is concerned, Bakmand54 has developed a microfluidic system adapted for cerebral tissue cultivation that combines conventional systems and fluid culture systems with the possibility of integrating a sensor system54. Primary neurons are grown on a modified peptide substrate (PNW) mimicking in vivo conditions54. It was found that tissues grown in the microfluidic system were superior in quality compared to those maintained on conventional plastic substrates54. As a support, biological membranes are used on which electrode sensors have been fixed to measure the nervous cell parameters in real-time 54 .

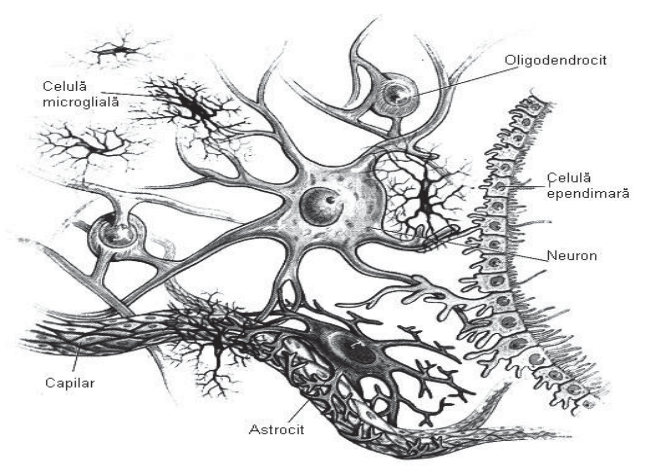

Figure 4 | Types of neuronal linkages in the structure of the nervous tissue (scheme). The neuron establishes links of the type of cell anastomoses with no electrical capacity (ependymal cells, capillary endothelium) or synapses (astrocytes, microglia, oligodendrocytes).

\section{Discussion}

Histologically, in the myocardium affected by cardiomyopathy, the disorganization of myofibrils occurs ${ }^{7}$. In the cytoplasm of myocardiocytes, myofibrillar subunits and neurofilaments may occur without Z-membrane organization and regular sarcomas, and in strongly dilated areas the myofibrillar disorganization and myofibrilolysis occur ${ }^{8}$. Different degrees of disorganization of actin and myosin filaments are observed in sarcomeres ${ }^{20}$. The nodal tissue is an essential element in the heart that initiates the contraction through the pacemaker cells from its structure ${ }^{21}$. Experimenting a method to improve its function and structure in dilated cardiomyopathy is vital in order to discuss about the success of treatment. In order to have clinical utility, cardioplasty using cardiospheres ${ }^{15}$, in vitro multiplied cardiac stem cells, requires a strategy that permits the activation, isolation, expansion 
and reintroduction into the cardiac lesion for the autologous therapy. More research is needed on the administration, the ability to differentiate and maintain a mature and functional cardiac phenotype, the mode of fusion with the host myocardium, the longevity of the grafts ${ }^{15}$, the risks and long-term side effects, especially immune responses and the rejection of the graft. Collectively, the studies previously presented on hiPSC systems ${ }^{18}$ show that chemical protocols have had notable scientific results but have been very little applied and are not suitable for largescale use. Among the successes, the patientspecific hiPSC derivatives allowed in vitro modeling of a large number of cardiovascular diseases and allowed the cardiotoxicity testing of some drugs ${ }^{18}$. By combining several experimental methods that have had notable success rates, each of them, but which could not be developed on large scale, I tend to think that the common approach presented in this study may increase the practical applicability rate of these implants.

\section{Conclusions}

The three-dimensional organization of cardiac stem cells and neurons couldimprove the contractile heart function and restore the main changes in dilatation cardiomyopathy. The concept of proliferation and differentiation of cardiac stem cells requires more in-depth studies in order to control these properties in vitro and in vivo.

HiPSC systems have an endless capacity for using; the challenge is to find exact specific phenotypes clinically adapted to each individual patient. More experimental research is needed to determine the degree of adaptability of the two types of cells in the receiving myocardium, the degree of improvement in the contractile function, and last but not least, the rate of postinterventional survival of affected dogs.

On the basis of the vital prognosis, a comparison can be made between the method presented in the study and the current symptomatic and adjuvant drug treatment.

\section{Bibliography}

1. Goodwin J.F. (1964), Cardiac function in primary myocardial disease, Editura Brit.Med.;

2. Zachary F. James, McGavin M. Donald (2011), Pathologic Basis of Veterinary Disease, Fifth Edition, Elsevier Ltd, Oxford;
3. Jubb, Kennedy and Palmer's (2015), Pathology of domestic animals, Sixth Edition, Elsevier Ltd, Oxford;

4. Dunn K. Jhon (1999), Textbook of small animal medicine, W.B. Saunders, Cambridge;

5. Done H. Stanley, Goody C. Peter, Evans A. Susan, Stickland C. Neil (2015), Color Atlas of Veterinary Anatomy, Second Edition, Vol. 3, Mosby Elsevier, London;

6. Ettinger J. Stephen, Feldman C. Edward, Cote Etienne (2011), Textbook of Veterinart Internal Medicine Expert Consult, Eith Edition, Saunders Elsevier, Canada;

7. Malvestio M.M. Lygia, Martins M. Isabela, Moares R. Flavio, Moraes R.E. Julieta (2015), Histopathologic evolution of cardiomyopathy in a canine model of Duchenne muscular dystrophy, Journal of Advanced Veterinary Research vol. 5: 121-126;

8. Janus I., Noszczyk-Nowak A., Nowak M., Ciaputa R., Kandefer-Gola M., Paslawska U. (2016), A comparison of the histopathologic pattern of the left atrium in canine dilated cardiomyopathy and chronic mitral valve disease, BMC Veterinary Research vol.12, no.3, ref.33;

9. Janus I., Noszczyk-Nowak A., Nowak M., Cepiel A., Ciaputa R., Paslawska U., Dzieqiel P., Jablonska K. (2014), Myocarditis in dogs: etiology, clinical and histopathological features. 11 cases (2007-2013), Irish Veterinary Journal vol. 67 , no. 28 , ref. 34 ;

10. Baisan A. Radu, Birsan Oana, Vulpe Vasile (2016), Electrocardiographic changes in chronic valvular disease and dilated cardiomyopathy in dog, Human \& Veterinary Medicine International Journal of the Bioflux Society vol.8: 98-102;

11. Olariu-Jurca A., Nichita Ileana, Stancu A., Ciulan V., Avram E., Lazau Al., Olariu-Jurca I. (2016), Morphological changes in noninflamatory cardiomyopathies in dogs, Lucrari Stiintifice Medicina Veterinara vol. XLIX(1): 136-141;

12. Cornell C. Craig, Kittleson D. Mark, Torre Della Paul, Haggstom Jens, Lombard W. Christophe, Pedersen D. Henrik, Vollmar Andreea, Wey Aaron (2004), Allometric scaling of M-mode cardiac measurements in normal adult dogs, Journal of Veterinary Internal Medicine vol.18: 311-321;

13. Giam Beverly, Chu Po-Yin, Kuruppu 
Sanjaya, Smith A. Ian, Horlock Duncan, Kiriazis Helen, Du Xiao-Jun, Kaye M. David, Rajapakse W.N. (2016), N-acelylcisteine attenuates the development of cardiac fibrosis and remodelling in a mouse model of heart failue, Physiological Reports vol. 4, Iss. 7;

14. Lisciandro R. Gregory (2016), The use of the diaphragmatico-hepatic views of the abdominal and thoracic focused assessment with sonography for triage examination for the detection of pericardial effusion in 24 dogs, Journal of Veterinary Emergency and Critical Care, no. 26: 125-131;

15. Barile Lucio, Chimenti Isotta, Gaetani Roberto, Forte Elvira, Miraldi Fabio, Frati Giacomo, Messina Elisa, Giacomello Alessandro (2007), Cardiac stem cells: isolation, expansion and experimental use for myocardial regeneration, Nature Clinical Practice, vol.4, sup. 1 ;

16. Starr Albert \& Co. (2007), The artificial heart valve, Nature Medicine, vol.13, no.10;

17. Zimarino Marco, D'Andreamatteo Mariangela, Waksman Ron, Epstein E. Stephen, Raffaele De Caterina (2014), The dynamics of the coronary collateral circulation, Nature Reviews Cardiology, vol.11;

18. Chen I. Yan, Matsa Elena, Wu C. Joseph (2016), Induced pluripotent stem cells: at the cardiovascular precision medicine, Nature Reviews Cardiology, vol.13;

19. Wislet-Gendebien S., Hans G., Leprince P., Rigo J.M., Moonen G., Rogister B. (2005), Plasticity of cultured mesenchymal stem cells: swich from nestin-positive to excitable neuronlike phenotype, Stem Cells Journals, vol.23, Iss.3:392-402;

20. Malik I. Fady, Hartman J. James, Elias A.K., Morgan P. Bradly, Rodriguez Hector, Brejc K., Anderson L. Robert, Sueoka H. Sandra, Lee H. K, Finer T. Jeffrey, Baliga Ramesh, Cox R. David, Godinez Guillermo, Kass A. David \& Co. (2011), Cardiac myosin activation a potential therapeutic approach for systolic heart failure, Science, vol. 331;

21. Kirk A. Jonathan, Chakir Khalid, Lee Han Kyoung, Karst Edward, Holewinski J. Ronald, Prionti Giangluigi, Tunnin S. Richard \&Co. (2015), Pacemaker-induced transient asynchrony suppresses heart failure progression, Science, vol.7, Iss. 319;

22. Bristow P., Sargent J., Fuentes V. Luis,
Brockman D. (2017), Outcome of bioprosthetic valve replacement in dogs with tricuspid valve dysplasia, Journal of Small Animal Practice, no. 58:205-210;

23. Urfer R. Silvan, Kaebrelin L. Tammi, Bergman J. Philip, Creevy E. Kate, Promislow L.E. Daniel, Kaebrelin Matt (2017), Asymptomatic heart valve dysfunction in healthy middle-aged companion dogs and its implications for cardiac aging, Springer Nature, vol.39, Iss.1:43-50;

24. Worlmald Dennis, Lawrence J.Andrew, Carter Gabrielle, Fisher D.Andrew (2017), Reduced heart rate variability in pet dogs affected by anxiety-related behaviour problems, Science, vol.168:122-127;

25. Markby G., Summers M.K., MacRae V.E., Del-Pozo J., Corcoran M.B. (2017), Myxomatous degeneration of the canine mitral valve: from gross changes to molecular events, Journal of Comparative Pathology, vol.156(4):371-383;

26. Harmon W. Mark, Leach B. Stacey, Lamb E. Kenneth (2017), Dilated cardiomyopathy in standard Schnauzers: retrospective study of 15 cases, Journal of the American Animal Hospital Association, vol.53, Iss.1:38-44;

27. Kawabata Atsushi, Husnik Roman, Le Donne Viviana, Grasperge Britton, Bordeaux L. Bonnie, Bauer Rudy (2017), Pathology in practice, Journal of the American Veterinary Medical Association, vol.205, no.10, 11131116;

28. Cullum P.A, Baiey J.S., Branfoot A.C., Pemberton M.J., Redding V.J., Rees J. Russell (2016), The warm ischaemic time of the canine heart, Cardiovascular Research, vol.4:67-72;

29. Vollmar A.C., Aupperle H. (2016), Cardiac pathology in Irish wolfhounds with heart disease, Journal of Veterinary Cardiology, vol 18(1): 57-70;

30. Lines Terje Glenn, Lino de Oliviera B., Skavhaug Ola, Maleckar M. Mary (2016), Simple T wave metrics may better predict early ischemia as compared to ST segment, IEEE Publication;

31. Shen L., Estrada A.H., Cote E., Powell M.A., Winter B., Lamb H. (2016), Aortoseptal angle and pressure gradient reduction following balloon valvuloplasty in dogs with severe subaortic stenosis, Journal of Veterinary Cardiology, vol. 19:144-152; 
32. Cepiel Alicja, Noszczyk-Nowak A. Paslawski Robert, Janiszewski Adrian, Paslawska Urszula (2017), Intracardiac electrophysiological conduction parameters in adult dogs, Veterinary Quarterly, vol 31:1, 9197;

33. Anversa P. \& Co. (2006), Life and death of cardiac stem cells: a paradigm shift in cardiac biology, Circulation 113: 1451-1463;

34. Nadin B.M. \& Co. (2003), Phenotype and hematopoietic potential of side population cells throughout embryonic development, Blood 102: 2436-2443;

35. Hierlihy A.M. \& Co. (2002), The post-natal heart contains a myocardial stem cell population, FEBS Lett 530: 239-243;

36. Martin C.M. \& Co. (2004), Persistent expression of the ATP-binding cassette transporter, Abcg2, identifies cardiac SP cells in the developing and adult heart, Dev Biol 265: 262-275;

37. Pfister O. \& Co. (2004), CD31- but not CD31+ cardiac side population cells exhibit functional cardiomyogenic differentiation, Circ Res 97: 52-61;

38. Beltrami A.P. \& Co. (2003), Adult cardiac stem cells are multipotent and support myocardial regeneration, Cell 114: 763-776;

39. Dawn B. \& Co. (2005), Cardiac stem cells delivered intravascularly traverse the vessel barrier, regenerate infarcted myocardium, and improve cardiac function, Proc Natl Acad Sci USA 102: 3766-3771;

40. Mouquet F. \& Co. (2005), Restoration of cardiac progenitor cells after myocardial infarction by self- proliferation and selective homing of bone marrow- derived stem cells, Circ Res 97: 1090-1092;

41. Rossi M.I. \& Co. (2005), Multicellular spheroids of bone marrow stromal cells: a threedimensional in vitro culture system for the study of hematopoietic cell migration, Braz J Med Biol Res 38: 1455-1462;

42. Mummery C. L. \& Co. (2012), Differentiation of human embryonic stem cells and induced pluripotent stem cells to cardiomyocytes: a methods overview, Circ. Res. 111, 344-358;

43. Mummery C. \& Co. (2003), Differentiation of human embryonic stem cells to cardiomyocytes: role of coculture with visceral endoderm-like cells, Circulation 107, 27332740;

44. Zwi L. \& Co. (2009), Cardiomyocyte differentiation of human induced pluripotent stem cells, Circulation 120, 1513-1523;

45. Zhang J. \& Co. (2009), Functional cardiomyocytes derived from human induced pluripotent stem cells, Circ. Res. 104, e30-e41; 46. Burridge P. W. \& Co. (2007), Improved human embryonic stem cell embryoid body homogeneity and cardiomyocyte differentiation from a novel V-96 plate aggregation system highlights interline variability, Stem Cells 25, 929-938;

47. Dubois N. C. \& Co. (2011), SIRPA is a specific cell-surface marker for isolating cardiomyocytes derived from human pluripotent stem cells, Nat. Biotechnol. 29, 1011-1018;

48. Laflamme M. A. \& Co. (2007), Cardiomyocytes derived from human embryonic stem cells in pro-survival factors enhance function of infarcted rat hearts, Nat. Biotechnol. 25, 1015-1024;

49. Zhang Q. \& Co. (2011), Direct differentiation of atrial and ventricular myocytes from human embryonic stem cells by alternating retinoid signals, Cell Res. 21, 579-587;

50. Elliott D.A.\&Co.(2011), NKX2-5eGFP/w hESCs for isolation of human cardiac progenitors and cardiomyocytes, Nat. Methods no. 8, 1037-1040;

51. Hudson J., Titmarsh D., Hidalgo A., Wolvetang E., Cooper-White J. (2012), Primitive cardiac cells from human embryonic stem cells, Stem Cells Dev. no.21, 1513-1523;

52. Lian X. \& Co. (2012), Robust cardiomyocyte differentiation from human pluripotent stem cells via temporal modulation of canonical Wnt signaling, Proc. Natl Acad. Sci. USA 109, E1848-E1857;

53. Burridge P. W. \& Co.(2014), Chemically defined generation of human cardiomyocytes, Nat. Methods 11, 855-860;

54. Bakmand T., Waagepetersen H. S. (2016), Micro fluidic system for culturing and monitoring of neuronal cells and tissue, DTU Nanotech; 